\title{
Tiling the Plane with a Fixed Number of Polyominoes
}

\author{
Nicolas Ollinger \\ Laboratoire d'informatique fondamentale de Marseille (LIF), \\ Aix-Marseille Université, CNRS, \\ 39 rue Joliot-Curie, 13013 Marseille, France, \\ Nicolas.0llinger@lif.univ-mrs.fr
}

\begin{abstract}
Deciding whether a finite set of polyominoes tiles the plane is undecidable by reduction from the Domino problem. In this paper, we prove that the problem remains undecidable if the set of instances is restricted to sets of 5 polyominoes. In the case of tiling by translations only, we prove that the problem is undecidable for sets of 11 polyominoes.
\end{abstract}

\section{Introduction}

Tiling the plane given a finite set of tiles is an old and fascinating problem. For an survey on tilings, the reader is invited to consult Grünbaum and Shephard [1]. A celebrated computability result by Berger [2] is the undecidability of the Domino problem: given a finite set of Wang tiles, unit squares with colored edges, decide if the Wang tiles can tile the whole plane so that matching edges share a same color. A polyomino is a simple kind of tile: it consists of rookwise connected unit squares. Golomb [3] studied tiling by polyominoes and proved in [4] that the Domino problem can be reduced to deciding if a finite set of polyominoes tiles the plane. The reduction can be achieved by a classical encoding of Wang tiles by polyominoes that preserves tilings. In this reduction, the number of polyominoes is equal to the initial number of Wang tiles. A natural question arises: what happens if we consider the tiling problem for a fixed number of polyominoes? From this previous result, two cases might happen: (1) the problem is undecidable starting from a certain fixed number of polyominoes (2) the problem is decidable for every fixed number of polyominoes but the family of decision procedures is not itself recursive. As case (1) is more likely to happen, the question is to find the frontier between decidability and undecidability. Such a study of decidability questions with respect to a parameter appears for example in the study of semi-Thue systems or for Post correspondence problem (PCP) where it is shown that $P C P(2)$ is decidable and $P C P(7)$ is undecidable [5-7].

Motivated by parallel computing, Wijshoff and van Leeuwen [8] proved that the tilability of the plane by translation of a unique polyomino is decidable. That result was further studied and understood by Beauquier and Nivat [9] who described precisely the tilings by translation generated by a unique polyomino.

This paper is organized as follows. In section 1, we introduce Wang tiles, polyominoes and dented polyominoes, a special variation of polyominoes used in 
the constructions. In section 2, we prove that it is undecidable whether a set of 5 polyominoes tiles the plane. In section 3 , we deduce from previous section that it is undecidable whether a set of 11 polyominoes tiles the plane by translation. In section 4 , we discuss the case of smaller sets of tiles.

\section{Definitions}

Polyominoes A polyomino is a simply connected tile obtained by gluing together rookwise connected unit squares. A tiling, of the Euclidian plane, by a set of polyominoes is a partition of the plane such that each element of the partition is the image by an isometry of a polyomino of the set. A tiling by translation is a tiling where isometries are restricted to translations. A tiling is periodic if it is invariant by translation, biperiodic if it is invariant by two non-colinear translations, aperiodic if it is not periodic. A set of polyominoes is aperiodic if it admits a tiling and all its tilings are aperiodic.

A tiling is discrete if all the vertices of the unit squares composing the polyominoes are aligned on the grid $\mathbb{Z}^{2}$. If a tiling is not discrete, the tiling can be split into two tilings of a half-plane along a line going through an edge along which two unit squares are not aligned. By shifting one half-plane to align the tiles, and iterating this process, one obtains the following lemma.

Lemma 1. A set of polyominoes admits a tiling if and only if it admits a discrete tiling.

In this paper where we deal with tilability, we only consider discrete tilings, thanks to this lemma. Thus, a polyomino can be considered as a finite, simply connected, subset of $\mathbb{Z}^{2}$ and a tiling by a set of polyominoes is a partition of $\mathbb{Z}^{2}$ where each element is the image by an isometry of an element of the set. Each such isometry can be decomposed into a translation and one out of 8 elementary transformations obtained by composing right angle rotations and mirroring. A sample polyomino and its 8 transformations are represented in Fig. 1.

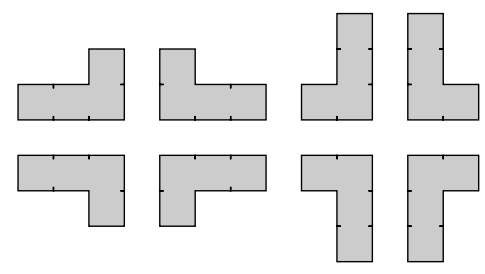

Fig. 1: A polyomino and its 8 transformations

Polyominoes are compactly described by their contour words. A contour word of a polyomino is a (finite) word on the alphabet $\{e, w, n, s\}$ describing a walk along the outline of the polyomino starting from and ending to a vertex of the 
boundary of the polyomino where $e$ is an east move $(1,0), w$ is a west move $(-1,0), n$ is a north move $(0,1)$ and $s$ is a south move $(0,-1)$. A word is a contour word if and only if the associated path does not cross itself. A polyomino with a pointed contour word is represented in Fig. 2

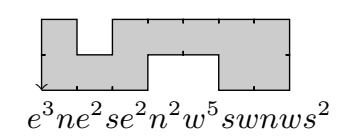

Fig. 2: A polyomino with a pointed contour word

Wang tiles A Wang tile is a unit square with colored edges. A tiling by a set of Wang tiles is a discrete tiling by tiles of the set such that along each edge the colors match on both sides. The Domino problem is the following decision problem: given a finite set of Wang tiles, decide whether it admits a tiling.

Theorem 1 (Berger [2]). The Domino problem is undecidable.

The Polyomino problem is the following decision problem: given a finite set of polyominoes, decide whether it admits a tiling. By a reduction from the Domino problem to the Polyomino problem, Golomb [4] proved the undecidability of the Polyomino problem.

Theorem 2 (Golomb [4]). The Polyomino problem is undecidable.

The reduction proceeds as follows. Given a finite set of Wang tiles, Golomb encodes each tile into a big squarish polyomino. Special bumps and dents are added to the corners of the tiles to force both alignment and orientation of the tiles: if one of the encoding polyominoes appears with an orientation, all the other tiles of the tiling have to use the same orientation. Special bumps and dents are used along the sides of the big polyominoes to encode the colors of the Wang tiles. Quotienting the set of tilings of the set of encoding polyominoes by isometries, it is in bijection with the set of tilings of the given set of Wang tiles.

Dented polyominoes A dented polyomino is a polyomino with edges labeled by a shape and an orientation. The four possible orientations $\{p, q, b, d\}$ and their interpretation depending on the direction of the edge are depicted on Tab. 1 for a sample shape. On a contour word, inside shapes define bumps and outside shapes define dents. A tiling by a set of dented polyominoes is a tiling by the corresponding set of polyominoes where bumps and dents match along edges.

Dented polyominoes provide a convenient tool to construct complicated sets of polyominoes. These polyominoes with puzzle bumps and dents can be easily converted into polyominoes. 


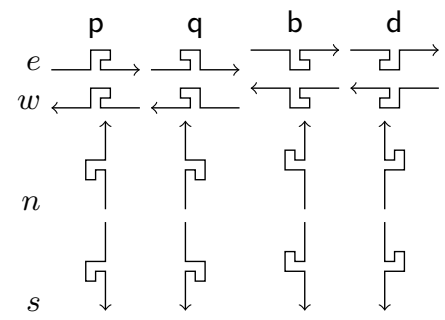

Table 1: Encoding of bumps and dents orientation

Lemma 2. Every finite set of dented polyominoes can be encoded as a finite set of polyominoes such that their sets of tilings are in one-to-one correspondence.

Proof. In order to guarantee that bumps and dents do not interfere with the matching conditions of polyominoes, the idea is to rescale the polyominoes. For all $k \in \mathbb{Z}^{+}$, a $k$-rescaling of a set of polyominoes consists into scaling the polyominoes by a factor $k$, i.e., replacing each unit square by a square of $k$ by $k$ unit squares. Tilings are preserved by rescaling: the set of tilings of a set of polyominoes is in one-to-one correspondence with the set of tilings of its $k$-rescaling.

To encode a finite set of dented polyominoes into a finite set of polyominoes: first, rescale the set of polyominoes by a factor far bigger than the size of any shape of its bumps and dents; then, add bumps and dents in the middle of each rescaled edge.

\section{Tiling with a fixed number of polyominoes}

The $k$-Polyomino problem is the following decision problem: given a set of $k$ polyominoes, decide whether it admits a tiling. This section is dedicated to the proof of the following theorem.

Theorem 3. The 5-Polyomino problem is undecidable.

We will proceed by reduction of the Domino problem. Given a finite set $\tau$ of Wang tiles, we construct a set of 5 dented polyominoes $P(\tau)$ such that, up to isometry, the set of tilings of $\tau$ is in one-to-one correspondence with the set of tilings of $P(\tau)$. The proof goes as follows. First, we describe the construction of $P(\tau)$. Then, we explain how to encode any tiling of $\tau$ by a tiling of $P(\tau)$. Finally, we show that any tiling of $P(\tau)$ encodes a tiling of $\tau$.

\subsection{Encoding a set of Wang tiles}

Let $\tau$ be a set of $N$ Wang tiles. The set of dented polyominoes $P(\tau)$ consists of the following 5 tiles, represented in Fig. 3:

meat encodes all tiles of the set $\tau$ sequentially; 
jaw acts as a selector to select exactly one tile of the meat;

filler is used for padding the blank leaved by the meat inside the jaw;

tooth erases the bits on the meat so that it fits inside the jaw;

wire links meat pieces together to verify tiling constraints.

More formally, the dented polyominoes use 4 different shapes for bumps and dents, detailed on Tab. 2.

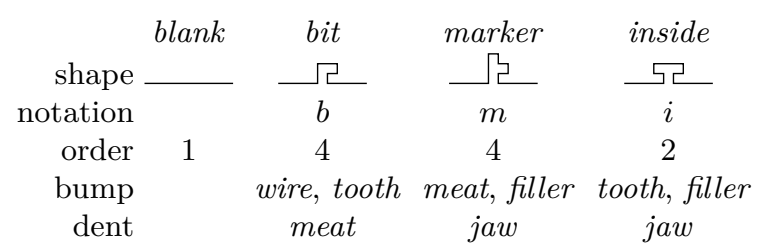

Table 2: Types of bumps and dents

Let $k$ be a large enough integer and choose an encoding on $k-4$ bits of the colors of the set of Wang tiles (horizontal and vertical colors can use different encodings). Let $\left(a_{j}^{i}\right),\left(b_{j}^{i}\right),\left(c_{j}^{i}\right),\left(d_{j}^{i}\right)$ be respectively the north, east, south, and west binary encoding of the tiles where $i$ is the tile index from 1 to $N$ and $j$ is the bit index from 1 to $k-4$. Let $\left(\mathfrak{a}_{j}^{i}\right)$ be the encoding of the $k$ bits, by adding prefix 00 and suffix 01 , of $\left(a_{j}^{i}\right)$ on the alphabet $\{\mathrm{b}, \mathrm{d}\}$. Let $\left(\mathfrak{b}_{j}^{i}\right)$ be the encoding of the $k$ bits, by adding prefix 10 and suffix 11, of $\left(b_{j}^{i}\right)$ on the alphabet $\{\mathrm{p}, \mathrm{q}\}$. Let $\left(\mathfrak{c}_{j}^{i}\right)$ be the encoding of the $k$ bits, by adding prefix 00 and suffix 01 , of $\left(c_{j}^{i}\right)$ on the alphabet $\{\mathrm{b}, \mathrm{d}\}$. Let $\left(\mathfrak{d}_{j}^{i}\right)$ be the encoding of the $k$ bits, by adding prefix 10 and suffix 11 , of $\left(d_{j}^{i}\right)$ on the alphabet $\{\mathrm{p}, \mathrm{q}\}$. The dented polyominoes are given by their contour words on Tab. 3 .

$$
\begin{aligned}
& \text { tooth: } e_{i}^{\mathrm{b}} n w_{b}^{\mathrm{q}} s \\
& \text { wire: } e_{b}^{\mathrm{b}} n^{5} w^{2 N(k+1)+1} n^{4} w_{b}^{\mathrm{p}} s^{5} e^{2 N(k+1)+1} s^{4} \\
& \text { filler: } e_{m}^{\mathrm{b}}\left(s e_{i}^{\mathrm{b}}\right)^{k}\left(e_{i}^{\mathrm{b}} n\right)^{k} e_{m}^{\mathrm{d}} n w_{m}^{\mathrm{q}}\left(n w_{i}^{\mathrm{p}}\right)^{k}\left(w_{i}^{\mathrm{p}} s\right)^{k} w_{m}^{\mathrm{p}} s \\
& \text { jaw: } \quad e_{m}^{\mathrm{q}}\left(e_{m}^{\mathrm{p}}\left(n e_{i}^{\mathrm{p}}\right)^{k}\left(e_{i}^{\mathrm{p}} s\right)^{k} e_{m}^{\mathrm{q}}\right)^{N-1} s\left(w_{m}^{\mathrm{d}}\left(s w_{i}^{\mathrm{d}}\right)^{k}\left(w_{i}^{\mathrm{d}} n\right)^{k} w_{m}^{\mathrm{b}}\right)^{N-1} w_{m}^{\mathrm{d}} s^{4} e^{2(N-1)(2 k+2)+4} n^{4} \\
& w_{m}^{\mathrm{b}}\left(w_{m}^{\mathrm{d}}\left(s w_{i}^{\mathrm{d}}\right)^{k}\left(w_{i}^{\mathrm{d}} n\right)^{k} w_{m}^{\mathrm{b}}\right)^{N-1} n\left(e_{m}^{\mathrm{p}}\left(n e_{i}^{\mathrm{p}}\right)^{k}\left(e_{i}^{\mathrm{p}} s\right)^{k} e_{m}^{\mathrm{q}}\right)^{N-1} e_{m}^{\mathrm{p}} n^{4} w^{2(N-1)(2 k+2)+4} s^{4} \\
& \text { meat: } \prod_{i=1}^{N}\left(e_{m}^{\mathrm{b}} \prod_{j=1}^{k-1}\left(e_{b}^{\mathfrak{a}_{j}^{i}} s\right) e_{b}^{\mathfrak{a}_{k}^{i}} \prod_{j=1}^{k-1}\left(e_{b}^{\mathfrak{b}_{j}^{i}} n\right) e_{b}^{\mathfrak{b}_{k}^{i}} e_{m}^{\mathrm{d}}\right) n \\
& \prod_{i=N}^{1}\left(w_{m}^{\mathrm{q}} \prod_{j=k}^{2}\left(w_{b}^{c_{j}^{i}} n\right) w_{b}^{\mathrm{c}^{i}} \prod_{j=k}^{2}\left(w_{b}^{\mathrm{o}_{j}^{i}} s\right) w_{b}^{\mathrm{o}^{i}} w_{m}^{\mathrm{p}}\right) s
\end{aligned}
$$

Table 3: Contour encoding of the tiles 


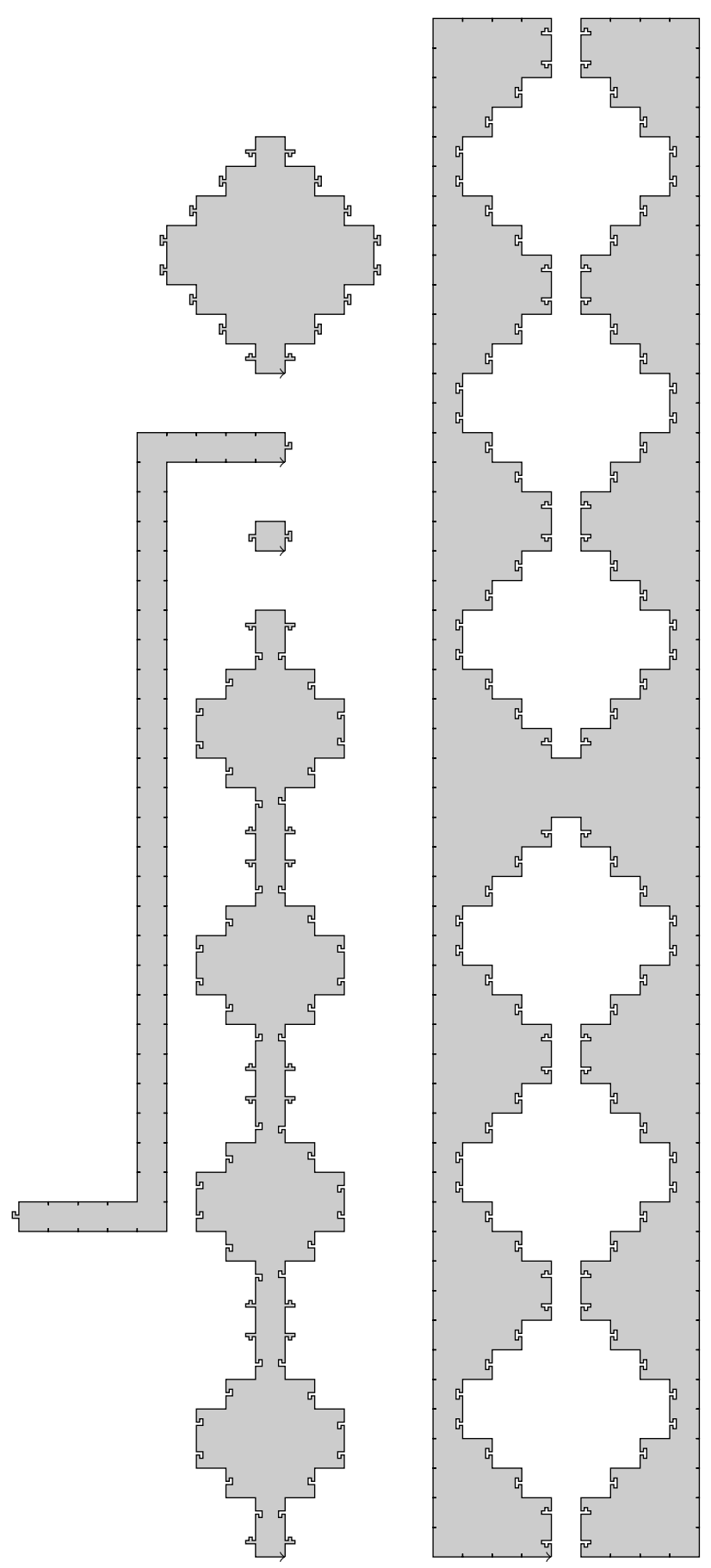

Fig. 3: Tiles (rotated to fit in page)

From left to right and bottom to top : meat, tooth, wire, filler, jaw

Notice that here $N=4$ and $k=3$ to fit the page (in the text $k>4$ ). 
Notice the following important properties of these tiles. The meat consists of a sequence of $N$ diamonds decorated with bit shapes with a prefix and a suffix marker shape pointing to the diamond. If one connects a tooth in each bit dent of a diamond of the meat, the diamond becomes similar to the filler. Moreover, both inside parts of a jaw consists of $N-1$ places to put a filler plus a marker shape at the entry of the jaw, pointing outside.

\subsection{Encoding tilings by Wang tiles}

Let us first prove the following lemma.

Lemma 3. Every tiling by $\tau$ can be encoded as a tiling by $P(\tau)$.

The set of dented polyominoes is designed to encode a Wang tile by selecting one diamond of a meat, hiding the other diamonds using two jaws on the left and the right, padding inside the jaws with teeth and fillers, as represented in Fig. 4. The colors of the tile are propagated to the four neighbor tiles using wires.

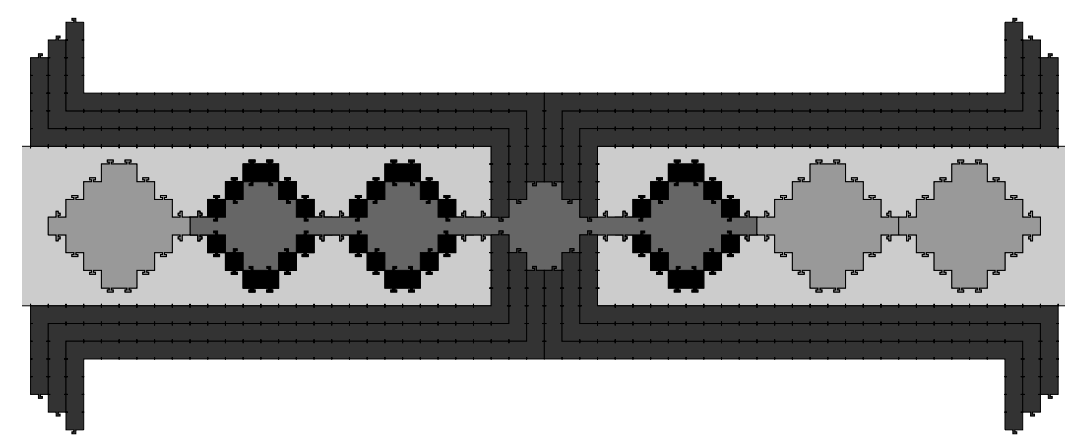

Fig. 4: Encoding of a Wang tile including inter-tiles wires

Let $T \in \tau^{\mathbb{Z}^{2}}$ be a tiling by $\tau$. Each north-west diagonal $(x+y=i$ for the $i$ th diagonal) is encoded as a line of Wang tile encodings where a jaw connects tile $T(x, i-x)$ to tile $T(x+1, i-x-1)$. These lines of encoding are put on top of each other with a slight translation so that tile $T(x, i-x)$ is connected by wires to $T(x+1, i-x), T(x, i-x+1), T(x-1, i-x)$ and $T(x, i-x-1)$, as represented in Fig. 5. Notice that the choices made for $\mathfrak{a}, \mathfrak{b}, \mathfrak{c}$, and $\mathfrak{d}$ permits such a connection only if the Wang tiling is valid. Thus, one obtains a tiling of the dented polyominoes.

\subsection{Every tiling encodes a tiling}

Now, we prove the following lemma. 


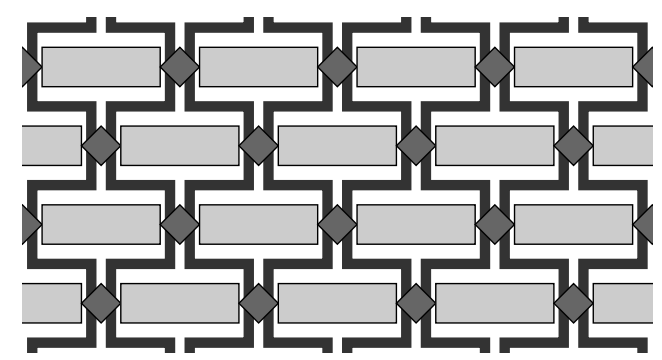

Fig. 5: Wiring of Wang tiles

Lemma 4. Every tiling by $P(\tau)$ encodes a tiling by $\tau$.

Consider a tiling by the dented polyominoes. We first show that it must contain a jaw. Consider any tile of the tiling. If it is a jaw, we are done. Examine Tab. 2. If it is a meat or a filler, it has a marker bump that should be linked to a dent only found on a jaw. If it is a tooth, it has an inside bump that should be linked to a dent only found on a jaw. Finally, if it is a wire, it has bit bump that should be linked to a meat, itself connected to a jaw.

Consider a jaw tile of the tiling. To fill all the marker bumps, only filler and meat tiles can be used. As fillers have inside bumps, they can only be used completely inside the jaw. Thus, the markers on the extremities of the jaw have to be filled by the dents of a meat. Consider the meat that fills a marker at the extremity of the jaw. As marker bumps only appear inside jaws, the marker dent on the other side of the diamond of the meat next to the jaw has to be at the extremity of a next jaw. The only possibility to fill the gap in between the jaw and the meat locking its extremity is to use fillers and teeth. By now, we have proved that each jaw of the tiling appears in a biinfinite line (or column if rotated) of alternating jaws and meats where each meat has exactly one selected diamond outside the jaws.

Consider now the diamond of each meat appearing outside the jaws: it has bit bumps. A bit dent is found only on teeth and wires. As a tooth cannot appear outside a jaw (it as an inside dent), only wires can be connected to these bumps. Consider the two bit bumps side by side at the top of the diamond hill. The only possibility for two wires to appear side by side is to have the left one point to the left and the right one point to the right. This enforces all the wires in between the jaw and the top hill wire to point in the same direction: left ones to the left and right ones to the right. Thus, every tiling by dented polyominoes consists of biinfinite lines (or columns) of selected meat diamonds connected by wires in a lattice way as on Fig. 5.

Due to isometries, it remains to prove that all the selected diamonds have the same orientation. This part is enforced by the prefix/suffix trick in the $\mathfrak{a}, \mathfrak{b}$, $\mathfrak{c}$, and $\mathfrak{d}$ encoding: the only possibility for a diamond to be connected to another diamond is that prefix and suffix match, thus both should be oriented in the same way. Thus, the tiling is the image by an isometry of a tiling by Wang tiles. 
We have proved that every tiling by $P(\tau)$ encodes a tiling by $\tau$, achieving the reduction.

\section{Tiling by translation}

The $k$-Polyomino translation problem is the following decision problem: given a set of $k$ polyominoes, decide if it admits a tiling by translation. Using the result of previous section, one obtains the following.

Theorem 4. The 11-Polyomino translation problem is undecidable.

To prove this corollary, for any finite set of Wang tiles $\tau$, construct a set of 11 polyominoes as follows. Consider the set of 5 dented polyominoes $P(\tau)$. To encode any tiling by $\tau$ into a tiling by $P(\tau)$ as done in the previous section, we use exactly:

1 transformation of meat;

1 transformation of jaw;

1 transformation of filler;

4 transformations of wire;

4 transformations of tooth.

The set of 11 polyominoes tiling by translation consists exactly of these tile transformations. These dented polyominoes admit a tiling if and only if the set of Wang tiles admits a tiling.

\section{Going further}

What can be said about tilability for sets of less that 5 polyominoes? or less than 11 polyominoes for tilings by translation? In the case of 1 polyomino, it is decidable for tiling by translation and still open for tiling with isometries.

Theorem 5 (Wijshoff and van Leeuwen [8], Gambini and Vuillon [10]). The 1-Polyomino translation problem is decidable in time quadratic in the size of the contour word.

\section{Open Problem 1. Is the 1-Polyomino problem decidable?}

To prove the undecidability of the Polyomino problem, one has to be able to construct aperiodic sets of polyominoes. Ammann et al provide a set of 2 polygonal tiles with colors and 3 polygonal tiles with bumps and dents that admits only aperiodic tilings. This set is convertible into polyominoes.

Theorem 6 (Ammann et al [11]). There exists an aperiodic set of 3 polyominoes.

Theorem 7 (Ammann et al [11]). There exists an aperiodic set of 8 polyominoes for tiling by translation.

Open Problem 2. Is the $k$-Polyomino problem decidable for $3 \leqslant k<5$ ?

Open Problem 3. Is the $k$-Polyomino translation problem decidable for $8 \leqslant k<11$ ? 


\section{Acknowledgement}

The author thanks Bruno Durand for challenging him with the decision problem of tiling the plane with a fixed number of polyominoes.

\section{References}

1. Grünbaum, B., Shephard, G.C.: Tilings and patterns. A Series of Books in the Mathematical Sciences. W. H. Freeman and Company, New York (1989)

2. Berger, R.: The undecidability of the domino problem. Memoirs American Mathematical Society 66 (1966)

3. Golomb, S.W.: Tiling with polyominoes. Journal of Combinatorial Theory 1(2) (1966) 280-296

4. Golomb, S.W.: Tiling with sets of polyominoes. Journal of Combinatorial Theory 9(1) (1970) 60-71

5. Post, E.L.: A variant of a recursively unsolvable problem. Bulletin of the American Mathematical Society 52 (1946) 264-268

6. Claus, V.: Some remarks on PCP $(\mathrm{k})$ and related problems. Bulletin of the EATCS 12 (1980) 54-61

7. Matiyasevich, Y., Sénizergues, G.: Decision problems for semi-thue systems with a few rules. Theoretical Computer Science 330(1) (2005) 145-169

8. Wijshoff, H.A.G., van Leeuwen, J.: Arbitrary versus periodic storage schemes and tessellations of the plane using one type of polyomino. Information and Control 62(1) (1984) 1-25

9. Beauquier, D., Nivat, M.: On translating one polyomino to tile the plane. Discrete and Computational Geometry 6(1) (1991) 575-592

10. Gambini, I., Vuillon, L.: An algorithm for deciding if a polyomino tiles the plane. Theoretical Informatics and Applications 41(2) (2007) 147-155

11. Ammann, R., Grünbaum, B., Shephard, G.: Aperiodic tiles. Discrete and Computational Geometry 8(1) (1992) 1-25 